\title{
A multi-institutional consensus on how to perform endoscopic ultrasound-guided peri-pancreatic fluid collection drainage and endoscopic necrosectomy
}

\author{
Jintao Guo, Adrian Saftoiu' ${ }^{1}$, Peter Vilmann², Pietro Fusaroli3, Marc Giovannini4, Girish Mishra5, \\ Surinder S. Rana ${ }^{6}$, Sammy Ho7, Jan-Werner Poley ${ }^{8}$, Tiing Leong Ang ${ }^{9}$, Evangelos Kalaitzakis ${ }^{10}$, \\ Ali A. Siddiqui ${ }^{11}$, Jose G. De La Mora-Levy ${ }^{12}$, Sundeep Lakhtakia ${ }^{13}$, Manoop S. Bhutani ${ }^{14}$, Malay Sharma ${ }^{15}$, \\ Shuntaro Mukai ${ }^{16}$, Pramod Kumar Garg ${ }^{17}$, Linda S. Lee ${ }^{18}$, Juan J. Vila ${ }^{19}$, Everson Artifon ${ }^{20}$, \\ Douglas G. Adler ${ }^{21}$, Siyu Sun
}

Endoscopy Center, Sheng Jing Hospital of China Medical University, Shenyang, China; ${ }^{1}$ Department of Endoscopy, University of Medicine and Pharmacy, Craiova, Romania; ${ }^{2}$ Endoscopy Department, GastroUnit, Copenhagen University Hospital, ${ }^{10}$ Endoscopy Unit, Digestive Disease Center, Copenhagen University Hospital, University of Copenhagen, Copenhagen, Denmark; ${ }^{3}$ Gastroenterology Unit, University of Bologna, Bologna, Italy; ${ }^{4}$ Pathology Unit (Flora Poizat), Institute Paoli-Calmettes, Marseille, France; ${ }^{5}$ Wake Forest Baptist Health, Winston-Salem, NC, ${ }^{7}$ Montefiore Medical Center, New York, ${ }^{11}$ Division of Digestive and Liver Diseases, Thomas Jefferson University Hospital, Philadelphia, PA, ${ }^{14}$ MD Anderson Cancer Center, University of Texas, Houston, TX, ${ }^{18}$ Division of Gastroenterology, Hepatology and Endoscopy, Brigham and Women's Hospital, Boston, MA, ${ }^{21}$ Division of Gastroenterology, University of Utah School of Medicine, Salt Lake City, UT, USA; ${ }^{6}$ Department of Gastroenterology, Postgraduate Institute of Medical Education and Research, Chandigarh, ${ }^{13}$ Department of Gastroenterology, Asian Institute of Gastroenterology, Hyderabad, Telangana, ${ }^{15}$ Department of Gastroenterology, Jaswant Rai Specialty Hospital, Meerut, Uttar Pradesh, ${ }^{17}$ Department of Gastroenterology, All India Institute of Medical Sciences, New Delhi, India; ${ }^{8}$ Erasmus MC University Medical Center Rotterdam, Rotterdam, The Netherlands; ' ${ }^{9}$ Gastroenterology Department, Changi General Hospital, Singapore, Singapore; ${ }^{12}$ Gastroenterology Division, National Cancer Institute, Mexico City, Mexico; ${ }^{16}$ Department of Gastroenterology and Hepatology, Tokyo Medical University, Tokyo, Japan; ${ }^{19}$ Endoscopy Unit, Complejo Hospitalario de Navarra, Pamplona, Spain; ${ }^{20}$ Department of Surgery, Ana Costa Hospital, Sao Paulo, Brazil

\section{ABSTRACT}

There is a lack of consensus on how endoscopic ultrasound (EUS)-guided pseudocyst drainage and endoscopic necrosectomy should be performed. This survey was carried out amongst members of the EUS Journal Editorial Board to describe their

\begin{tabular}{|l|l|}
\hline \multicolumn{2}{|c|}{ Access this article online } \\
\hline Quick Response Code: & Website: \\
\hline & www.eusjournal.com \\
\cline { 2 - 2 } & DOI: \\
\hline
\end{tabular}

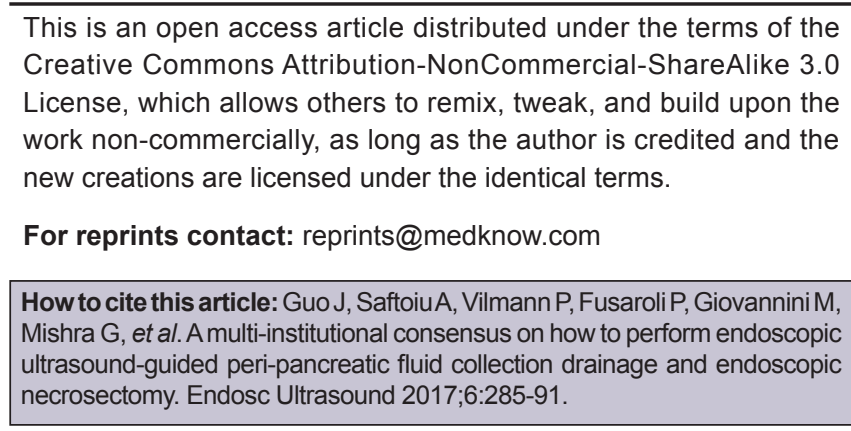

\section{Address for correspondence}

Prof. Siyu Sun, Endoscopy Center, Shengjing Hospital of China Medical University, No. 36, Sanhao Street, Shenyang 110004 , Liaoning Province, China. E-mail: sunsy@sj-hospital.org

Prof. Douglas G. Adler, Department of Gastroenterology and Hepatology, Huntsman Cancer Center, University of Utah School of Medicine, 30N 1900E 4R118, Salt Lake City, UT 84132, USA. E-mail: douglas.adler@hsc.utah. edu

Received: 2017-07-16; Accepted: 2017-09-12 
practices in performing this procedure. This was a worldwide multi-institutional survey amongst members of the EUS Journal Editorial Board in May 2017. The responses to a 22-question survey with respect to the practice of EUS-guided pseudocyst drainage and endoscopic necrosectomy were obtained. Twenty-two endoscopists responded to the questionnaire as follows: $72.7 \%(16 / 22)$ were of the opinion that lumen-apposing metal stents (LAMS) should be the standard of care for the creation of an endoscopic cystenterostomy in patients with pancreatic walled-off necrosis (WON); 95.5\% (21/22) recommended large diameter $(\mathrm{d}=15 \mathrm{~mm})$ LAMS for drainage in patients with WON; $54.5 \%(12 / 22)$ would not dilate LAMS after placement into the WOPN; 86.4\% (19/22) would not perform endoscopic necrosectomy during the same procedure as the creation of the cystenterostomy; $45.5 \%$ (10/22) recommend that agents, such as diluted hydrogen peroxide, should be used to lavage the peripancreatic fluid collection (PFC) cavity in patients with WON; and 45.5\% (10/22) considered a naso-cystic or other tube to be necessary for lavage of WON after initial drainage. The mean optimal interval recommended for endoscopic necrosectomy procedures after EUS-guided drainage was 6.23 days. The mean optimal interval recommended for repeat imaging in patients undergoing endoscopic necrosectomy was 12.32 days. The mean time recommended for LAMS removal was 4.59 weeks. This is the first worldwide survey on the practice of EUS-guided pseudocyst drainage and endoscopic necrosectomy. There were wide variations in practice and randomized studies are urgently needed to establish the best approach for management of this condition. There is also a pressing need to establish a best practice consensus.

Key words: consensus; endoscopic necrosectomy; endoscopic ultrasound; peri-pancreatic fluid collection

\section{INTRODUCTION}

Peri-pancreatic fluid collections (PFCs) frequently occur in the context of acute and chronic pancreatitis. Severe cases of pancreatitis, independent of the underlying cause, can lead to the development of PFCs. ${ }^{[1]}$ Endoscopic ultrasound (EUS)-guided drainage of a PFC is a minimally invasive procedure that is widely used, and in many institutions worldwide, EUS-guided drainage of a PFC is standard therapy for pancreatic pseudocysts (PPs) and pancreatic walled-off necrosis (WON). ${ }^{[2-5]}$ The advent and widespread dissemination of lumen-apposing metal stents (LAMS) as a tool in EUS have been reported to be successful in the management of PFCs. ${ }^{[6,7]}$ Endoscopic necrosectomy can supplant and replace surgical necrosectomy in the vast majority of patients. Endoscopic necrosectomy is minimally invasive, does not require an abdominal incision, and allows debridement and clearance of very large amounts of necrotic tissue. There is a lack of agreement and consensus on how EUS-guided peri-pancreatic fluid collection drainage and endoscopic necrosectomy should be performed. ${ }^{[8]}$ We present a survey of expert endoscopists regarding endoscopic drainage and necrosectomy techniques to assess practice patterns worldwide.

\section{PATIENTS AND METHODS}

A 22-question survey regarding the practice of EUS-guided PFC drainage and endoscopic necrosectomy was created and disseminated to members of the EUS Journal Editorial Board in May 2017. Before completing the survey, the participants were reminded that the questions listed were only directed toward EUS-guided drainage and subsequent endoscopic necrosectomy of WON or pancreatic abscesses. The responses to the questions were reported anonymously.

\section{Design of the questionnaire}

The questions in the questionnaire were grouped under several sections, including the type of stent used for drainage, the diameter of the stent, the necrosectomy procedure, endoscopic tools utilized for necrosectomy, and postprocedural management. Because no international guidelines or consensus for the procedure exist, the formulation of questions used was based on the opinions of regional experts regarding what constitutes the most important aspects or controversial areas of EUS-guided peri-pancreatic fluid collection drainage and endoscopic necrosectomy. Statements were formulated by combining a formal literature review of the endoscopic treatment of WON with expert opinions from the members of the EUS Journal Editorial Board [Table 1].

\section{Statistical analysis}

Continuous variables are reported as the mean \pm standard deviation (SD) or median and range when the distribution is highly skewed. Categorical variables are summarized with frequencies and proportions. Statistical analyses were performed using SPSS ${ }^{\circledR} 22.0$ statistical software (SPSS, Inc., Chicago, IL, USA).

\section{RESULTS}

Advances in endoscopic tools have driven a new era of minimally invasive techniques to manage PPs and 
Guo, et al.: Consensus of endoscopic ultrasound-guided pseudocyst drainage and endoscopic necrosectomy

\begin{tabular}{|c|c|}
\hline $\begin{array}{l}\text { Category and } \\
\text { grade }\end{array}$ & Description \\
\hline \multicolumn{2}{|c|}{ Quality of evidence } \\
\hline 1 & $\begin{array}{l}\text { Evidence obtained from } \\
\text { at least one RCT }\end{array}$ \\
\hline$\|-1$ & $\begin{array}{l}\text { Evidence obtained from well-designed } \\
\text { control trials without randomization }\end{array}$ \\
\hline II-2 & $\begin{array}{l}\text { Evidence obtained from a well-designed } \\
\text { cohort or case-control study }\end{array}$ \\
\hline II-3 & $\begin{array}{l}\text { Evidence obtained from comparisons } \\
\text { between times or places with } \\
\text { or without intervention }\end{array}$ \\
\hline III & $\begin{array}{l}\text { Opinion of respected authorities } \\
\text { based on clinical experience } \\
\text { and expert committees }\end{array}$ \\
\hline \multicolumn{2}{|c|}{$\begin{array}{l}\text { Classification of the } \\
\text { recommendation }\end{array}$} \\
\hline$A$ & $\begin{array}{l}\text { There is good evidence to } \\
\text { support the statement }\end{array}$ \\
\hline B & $\begin{array}{l}\text { There is fair evidence to } \\
\text { support the statement }\end{array}$ \\
\hline C & $\begin{array}{l}\text { There is poor evidence to support the } \\
\text { statement, but the recommendation } \\
\text { was made on other grounds }\end{array}$ \\
\hline D & $\begin{array}{l}\text { There is fair evidence to } \\
\text { refute the statement }\end{array}$ \\
\hline $\mathrm{E}$ & $\begin{array}{l}\text { There is good evidence to } \\
\text { refute the statement }\end{array}$ \\
\hline \multicolumn{2}{|c|}{$\begin{array}{l}\text { Voting on the } \\
\text { recommendation }\end{array}$} \\
\hline$A$ & Accept completely \\
\hline B & Accept with some reservations \\
\hline C & Accept with major reservations \\
\hline $\mathrm{D}$ & Reject with reservations \\
\hline $\mathrm{E}$ & Reject completely \\
\hline
\end{tabular}

RCT: Randomized controlled trial

WON. ${ }^{[2-5]}$ The conventional single transluminal gateway technique using transmural placement of single or multiple plastic or metal stents is sufficient for the vast majority of PFCs. ${ }^{[9]}$ The primary liquid content of PFCs can be easily drained with a single, transmural drain to allow for collapse and resolution; however, the solid necrotic tissue contained within a WON often does not drain as easily, which may impair the outcome of endoscopic drainage by leading to premature stent occlusion with the potential to develop infection. Furthermore, infected WON requires aggressive therapy, such as direct endoscopic necrosectomy (DEN), for the removal of necrotic tissue. ${ }^{[2-5]}$

\section{Question 1}

Should LAMS become the standard of care for the creation of an endoscopic cystenterostomy in patients with pancreatic necrosis?

\section{Answer}

The majority of endoscopists $(72.7 \%$ [16/22]) felt that LAMS should become the standard of care for creation of an endoscopic cystenterostomy in patients with pancreatic WON.

Although LAMS are increasingly being used for drainage of WON, the advantage over plastic stents is unclear. The current survey suggests that endoscopic transmural drainage using metal stents, such as LAMS and DEN, is widely perceived to be effective and safe. LAMS were developed with the aim of reducing the rate of migration and has a dog bone shape with a double flange, thus allowing apposition of the walls of the cavity and enteric wall. The wide diameter enables the passage of an endoscope for necrosectomy. Plastic stents have the disadvantage of a small lumen diameter, which can limit drainage and may necessitate reintervention, which have to be removed in most cases to allow necrosectomy to proceed. When the stents are removed, there is a risk of loss of access to the PFC cavity, whereas necrosectomy can occur through the LAMS with much less risk of loss of access. Mukai et al. ${ }^{[9]}$ retrospectively evaluated the safety, efficacy, and cost performance of draining WON using a novel, fully covered, biflanged metal stent (BFMS) compared with a traditional plastic stent. Seventy patients with symptomatic WON were treated under EUS guidance. Initial drainage was conducted using the single gateway technique with placement of one or more plastic stents or a single BFMS. If drainage alone was unsuccessful, DEN was performed. Plastic stents and BFMS were safe and effective for the treatment of WON. In particular, BFMS placement appeared to be preferable for initial EUS-guided drainage and additional DEN as it reduced the procedure time.

Another multicenter study also confirmed the effectiveness of LAMS in the treatment of a WON. Sharaiha et al. ${ }^{[6]}$ studied 124 patients with WON who underwent endoscopic transmural drainage using LAMS at 17 tertiary care centers from January 2014 to May 2015. After EUS-guided drainage, endoscopic necrosectomy was performed directly, irrigated with hydrogen peroxide $\left(\mathrm{H}_{2} \mathrm{O}_{2}\right)$, and/or a nasocystic drain was placed. The median size of the WON was $9.5 \mathrm{~cm}$ (range, $4-30 \mathrm{~cm}$ ). Eight patients had 2 LAMS placed for multiport access, all with technical success $(100 \%)$. Clinical success was achieved in 107 patients (86.3\%) after 3 months of follow-up. The stents remained patent in $94 \%$ of the patients 
Guo, et al:: Consensus of endoscopic ultrasound-guided pseudocyst drainage and endoscopic necrosectomy

(117 of 124) and migrated in $5.6 \%$ of the patients (7 of 124). In this questionnaire, most endoscopists felt that LAMS should be the standard of care for the creation of an endoscopic cystenterostomy in patients with a pancreatic WON.

Quality of evidence: II-1.

Classification of recommendation: B.

Level of agreement: (a) $72.7 \%$, (b) $22.7 \%$, (c) $4.6 \%$, (d) $0 \%$, (e) $0 \%$.

\section{Question 2}

What size of LAMS is optimal in patients with pancreatic necrosis?

\section{Answer}

Twenty-one of $22(95.5 \%)$ endoscopists recommended a large diameter $(\mathrm{d}=15 \mathrm{~mm})$ LAMS for drainage in patients with WON.

What size of LAMS is optimal in patients with pancreatic necrosis? In this survey, 95.5\% (21/22) of endoscopists felt that a large diameter $(\mathrm{d}=15 \mathrm{~mm})$ LAMS for the drainage of WON is more effective. Larger diameter LAMS are commercially available in Europe at this time, of note. It would have been of interest if Lakhtakia et al. ${ }^{[10]}$ had performed a subgroup analysis to determine whether patients who had a smaller diameter stent required more procedures for endoscopic declogging or a higher number of DEN sessions compared with a larger stent diameter. Because the larger stent diameter is one of the key reasons for success of the BFMS and LAMS in the management of WON, future devices will likely have stents with even larger diameters.

Will application of different stent treatments for WON have different results? Siddiqui et al. ${ }^{[7]}$ compared the clinical outcomes and adverse events of EUS-guided drainage/debridement of WON with double-pigtail plastic (DP) stents, fully covered, self-expanding metal stents (FCSEMSs), and LAMS. From 2010 to 2015, a total of 313 patients $(23.3 \%$ female; mean age, 53 years) underwent WON debridement, including 106 who were drained using DP stents, 121 using FCSEMSs, and 86 using LAMS. Successful endoscopic therapy was noted in 277 of $313(89.6 \%)$ patients. These authors concluded that EUS-guided drainage/debridement of WON using
FCSEMSs and LAMS is superior to DP stents with respect to overall treatment efficacy. The number of procedures required for WON resolution was significantly lower with LAMS compared with FCSEMSs and DP stents. Some novel stents are also used in the treatment of WON.

Some novel metal stents are increasingly used in the treatment of WON. Huggett et al. ${ }^{[11]}$ used a novel FCSEMS (NAGI stent; Taewoong Medical, Gyeonggi-do, Korea) for EUS-guided drainage of WON. Stents with a $14-\mathrm{mm}$ or 16-mm expanded diameter and 20 - or $30-\mathrm{mm}$ length were used. Nineteen patients were included. The median maximum collection size was $15 \mathrm{~cm}$, with a median necrosis rate of $50 \%$. Fourteen of $19(73.7 \%)$ patients underwent necrosectomy. Five stents migrated or dislodged. One patient had postprocedure abdominal pain. The conclusion of the Huggett et al.'s ${ }^{[1]}$ study was that the novel FCSEMS is feasible and safe for drainage of WON. The same metal stent also used in the Mukai et al.'s ${ }^{[12]}$ study. Twenty-one patients were treated by EUS-guided drainage using a flared-type BFMS for PFCs (PP, 2 patients; WON, 19 patients). The present study showed a technical success rate of $100 \%$ and a final clinical success rate of $100 \%$.

Quality of evidence: II-1.

Classification of recommendation: B.

Level of agreement: (a) $95.5 \%$, (b) $4.5 \%$, (c) $0 \%$, (d) $0 \%$, (e) $0 \%$.

\section{Question 3}

Should LAMS be dilated after deployment or allowed to open on its own after placement?

\section{Answer}

Twelve of $22(54.5 \%)$ endoscopists recommended that LAMS should not be dilated after placement into the WON.

Twelve of $22(54.5 \%)$ endoscopists considered that the LAMS should not be dilated after placement into the WON. If the diameter of the stent is large enough, dilation is unnecessary. Dilation may facilitate more rapid drainage and may help to bring the PFC into closer apposition with the gastric or duodenal wall, but there is a risk of dislodgment after deployment. It is 
likely that some endoscopists dilate in some cases, and not in other cases. Whether the endoscopists choose to dilate or not is always based on the content and appearance of PFC.

Quality of evidence: III.

Classification of recommendation: C.

Level of agreement: (a) $36.5 \%$, (b) $0 \%$, (c) $4.5 \%$, (d) $4.5 \%$, (e) $54.5 \%$.

\section{Question 4}

Should endoscopic necrosectomy be performed during the same procedure as the creation of the cystenterostomy? What is the optimal interval for repeat endoscopic necrosectomy procedures?

\section{Answer}

Nineteen of $22(86.4 \%)$ endoscopists were of the opinion that endoscopic necrosectomy should not be performed during the same procedure as the creation of the cystenterostomy. The mean $( \pm S D$ ) optimal interval recommended for endoscopic necrosectomy procedures after EUS-guided drainage was $6.23 \pm 4.71$ days (range, 3-21 days).

Gornals et al. ${ }^{[13]}$ prospectively recruited patients with a WON after necrotizing pancreatitis who underwent endoscopic transmural necrosectomy using LAMS with vigorous irrigation sessions. After drainage by LAMS, irrigation necrosectomy sessions were planned at intervals of $2-5$ days until most of the nonadherent necrotic material was extruded, and there was clinical improvement. Endoscopic necrosectomy was performed on the same day in several cases. All stents were successfully positioned in all cases, which resulted in a technical success rate of $100 \%$. Resolution of the lesions was achieved in $100 \%$ of cases after a median of three endoscopy sessions per patient.

In the survey, most endoscopists were of the opinion that endoscopic necrosectomy should not be performed during the same procedure as the creation of a cystenterostomy, although those that do so cannot be seen as violating the standard of care. The mean optimal interval recommended for endoscopic necrosectomy procedures after EUS-guided drainage was considered to be 6.23 days (range, 3-21 days). After fistula formation, the pneumoperitoneum and bleeding complication rates were lower.
Quality of evidence: II-1.

Classification of recommendation: B.

Level of agreement: (a) $13.6 \%$, (b) $0 \%$, (c) $0 \%$, (d) $0 \%$, (e) $86.4 \%$.

\section{Question 5}

Should a nasocystic tube (or other tube) be needed? Should agents, such as diluted $\mathrm{H}_{2} \mathrm{O}_{2}$, be used universally to lavage the PFC cavity in patients with pancreatic necrosis?

\section{Answer}

Ten of $22(45.5 \%)$ endoscopists were of the opinion that a nasocystic or other tube would be needed to lavage the PFC cavity. 45.5\% (10/22) recommended that agents such as diluted $\mathrm{H}_{2} \mathrm{O}_{2}$, should be used universally to lavage the PFC cavity in patients with a WON.

In a retrospective study, the use of a nasocystic tube alongside PSs in patients with a WON was shown to result in higher short-term success ( $85 \%$ vs. $63 \%$ ) and decreased stent occlusion rates (13\% vs. 33\%) compared to the use of PS alone. ${ }^{[14]}$ Furthermore, a recently published Cochrane review concluded that the use of a nasocystic tube with EUS-guided drainage was associated with lower adverse events and a shorter hospital stay than when EUS-guided drainage was performed alone. ${ }^{[15]}$ Lakhtakia et al. ${ }^{[10]}$ retrospectively evaluated consecutive patients with symptomatic WON who underwent EUS-guided drainage using BFMSs over a 3 -year period. Two hundred and five WON patients were included. Reassessment was performed between 48 and $72 \mathrm{~h}$ for resolution. Endoscopic reinterventions were tailored in nonresponders in a stepwise manner. Step 1: Occluding debris was cleared using endoscopic devices (declogging) using a snare or forceps. Step 2: Nasocystic tube (NCT) placement through BFMSs with intermittent irrigation. Step 3: DEN. Three days after the BFMS was placed for drainage, a gastroscopic assessment was performed to confirm stent dysfunction. As the first step in this approach, any occluding debris was cleared with endoscopic devices (declogging) using a snare or forceps. In symptomatic patients with necrotic debris visible within the WON cavity, but without stent occlusion, and in patients who did not improve with the step one approach (declogging), the second step was to place an NCT through the lumen of the BFMS. 
Guo, et al.: Consensus of endoscopic ultrasound-guided pseudocyst drainage and endoscopic necrosectomy

Intermittent irrigation with saline solution and $3 \% \mathrm{H}_{2} \mathrm{O}_{2}$ was then undertaken. Each session of irrigation was done at 8-hourly intervals using $20 \mathrm{~mL}$ of $3 \% \mathrm{H}_{2} \mathrm{O}_{2}$, followed $10 \mathrm{~min}$ later by $100 \mathrm{~mL}$ of saline solution. Sharaiha et al. ${ }^{[6]}$ conducted a retrospective multicenter case series of 124 with WONs who underwent endoscopic transmural drainage using LAMS (Axios; Boston Scientific, Natick, Massachusetts, USA) at 17 tertiary care centers from January 2014 to May 2015. Patients underwent EUS-guided cystogastrostomy or cystenterostomy with placement of LAMS into the WON collection. At the discretion of the endoscopist, direct endoscopic necrosectomy, irrigation with $\mathrm{H} 2 \mathrm{O} 2$, and/or a NCT drain placement was performed. Clinical success was achieved in 107 patients (86.3\%) after 3 months of follow-up.

Quality of evidence: II-1.

Classification of recommendation: $\mathrm{B}$.

Level of agreement: (a) $45.5 \%$, (b) $22.7 \%$, (c) $18.2 \%$, (d) $13.6 \%$, (e) $0 \%$.

\section{Question 6}

What is the optimal interval for repeat imaging in patients undergoing endoscopic necrosectomy? When should LAMS or other stents be removed in patients undergoing endoscopic necrosectomy?

Answer

The mean $( \pm S D)$ optimal interval recommended for repeat imaging in patients undergoing endoscopic necrosectomy was $12.32 \pm 7.46$ days (range, 3-30 days). The mean ( \pm SD) time recommended to remove the LAMS was $4.59 \pm 1.919$ weeks (range, 3-12 weeks).

Gornals et al. ${ }^{[13]}$ reported that WON resolution was assessed by multidetector computed tomography or EUS 4-8 weeks after initial transmural drainage. If complete resolution was achieved, all stents were removed; however, if the collection was not resolved, stent removal was deferred and a new imaging procedure was scheduled 2-4 weeks later.

The use of LAMS for PFCs has been previously reported. ${ }^{[16-20]}$ High complication rates related to migration have also been documented. ${ }^{[17,21]}$ When to remove the stent remains controversial and will likely remain individualized. Long-term rates of stent patency and safety are unknown and require further investigation. In the survey, the mean time recommended to remove the LAMS was 4.59 weeks (range, 3-12 weeks). Hence, follow-up is important for patients with a LAMS, specifically with respect to postprocedural stent migration. Gornals et al. ${ }^{[13]}$ reported that all stents were easily retrievable after resolution of the lesions. The mean time to stent retrieval was $9 \pm 3.4$ weeks without stent migration. Lakhtakia et al. ${ }^{[10]}$ reported that all of the patients were followed for 4-8 weeks, at which time the BFMS was removed after documenting resolution of the WON on transabdominal ultrasound imaging.

Quality of evidence: II-2.

Classification of recommendation: C.

\section{CONCLUSION}

Based on the consensus, most endoscopists recommended that LAMS should be the standard of care for the creation of an endoscopic cystenterostomy in patients with pancreatic WONs. A large diameter $(\mathrm{d}=15 \mathrm{~mm})$ LAMS are more effective for drainage and following endoscopic necrosectomy. The majority of endoscopists felt that endoscopic necrosectomy should not be performed during the same procedure as the creation of a cystenterostomy. The mean optimal interval recommended for endoscopic necrosectomy procedures after EUS-guided drainage is 6.23 days. To avoid stent migration or hemorrhage caused by the LAMS, the mean time recommended to remove the LAMS is 4.59 weeks. Whether or not, a plastic stent should be placed after removal of the LAMS was not addressed. Similarly, the issue of transpapillary pancreatic duct (PD) stenting by ERCP in addition to transmural drainage was not addressed in this survey. There is a need for RCTs to compare metal with plastic stents in pseudocyst due to acute pancreatitis and WON separately to decrease heterogeneity. Pseudocyst associated with chronic pancreatitis should be dealt with differently because there is hardly any necrosis in them thus obviating the need for a metal stent. However, patients with chronic pancreatitis may require $\mathrm{PD}$ stenting due to $\mathrm{PD}$ obstruction and communication with the cyst.

\section{Financial support and sponsorship}

Nil. 
Guo, et al:: Consensus of endoscopic ultrasound-guided pseudocyst drainage and endoscopic necrosectomy

\section{Conflicts of interest}

There are no conflicts of interest.

\section{REFERENCES}

1. Tyberg A, Karia K, Gabr M, et al. Management of pancreatic fluid collections: A comprehensive review of the literature. World J Gastroenterol 2016;22:2256-70.

2. Braden B, Dietrich CF. Endoscopic ultrasonography-guided endoscopic treatment of pancreatic pseudocysts and walled-off necrosis: New technical developments. World J Gastroenterol 2014;20:16191-6.

3. Capone P, Petrone MC, Dabizzi E, et al. Endoscopic ultrasound-guided drainage of a pancreatic fluid collection using a novel lumen-apposing metal stent complicated by stent occlusion. Endoscopy 2016;48 Suppl 1:E203.

4. Singhal S, Rotman SR, Gaidhane M, et al. Pancreatic fluid collection drainage by endoscopic ultrasound: An update. Clin Endosc 2013;46:506-14.

5. Alali A, Mosko J, May G, et al. Endoscopic ultrasound-guided management of pancreatic fluid collections: Update and review of the literature. Clin Endosc 2017;50:117-25.

6. Sharaiha RZ, Tyberg A, Khashab MA, et al. Endoscopic therapy with lumen-apposing metal stents is safe and effective for patients with pancreatic walled-off necrosis. Clin Gastroenterol Hepatol 2016;14:1797-803.

7. Siddiqui AA, Kowalski TE, Loren DE, et al. Fully covered self-expanding metal stents versus lumen-apposing fully covered self-expanding metal stent versus plastic stents for endoscopic drainage of pancreatic walled-off necrosis: Clinical outcomes and success. Gastrointest Endosc 2017;85:758-65.

8. Adler DG, Siddiqui AA. Nobody really knows how to perform endoscopic necrosectomy. Endosc Ultrasound 2017;6:147-8

9. Mukai S, Itoi T, Baron TH, et al. Endoscopic ultrasound-guided placement of plastic vs. biflanged metal stents for therapy of walled-off necrosis: A retrospective single-center series. Endoscopy 2015;47:47-55.
10. Lakhtakia S, Basha J, Talukdar R, et al. Endoscopic "step-up approach" using a dedicated biflanged metal stent reduces the need for direct necrosectomy in walled-off necrosis (with videos). Gastrointest Endosc 2017;85:1243-52.

11. Huggett MT, Oppong KW, Pereira SP, et al. Endoscopic drainage of walled-off pancreatic necrosis using a novel self-expanding metal stent. Endoscopy 2015;47:929-32.

12. Mukai S, Itoi T, Sofuni A, et al. Clinical evaluation of endoscopic ultrasonography-guided drainage using a novel flared-type biflanged metal stent for pancreatic fluid collection. Endosc Ultrasound 2015;4:120-5.

13. Gornals JB, Consiglieri CF, Busquets J, et al. Endoscopic necrosectomy of walled-off pancreatic necrosis using a lumen-apposing metal stent and irrigation technique. Surg Endosc 2016;30:2592-602.

14. Siddiqui AA, Dewitt JM, Strongin A, et al. Outcomes of EUS-guided drainage of debris-containing pancreatic pseudocysts by using combined endoprosthesis and a nasocystic drain. Gastrointest Endosc 2013;78:589-95.

15. Gurusamy KS, Pallari E, Hawkins N, et al. Management strategies for pancreatic pseudocysts. Cochrane Database Syst Rev 2016;4:CD011392.

16. Talreja JP, Shami VM, Ku J, et al. Transenteric drainage of pancreatic-fluid collections with fully covered self-expanding metallic stents (with video). Gastrointest Endosc 2008;68:1199-203.

17. Belle S, Collet P, Post S, et al. Temporary cystogastrostomy with self-expanding metallic stents for pancreatic necrosis. Endoscopy 2010;42:493-5.

18. Adler DG, Siddiqui AA. What's in a name? Pancreatic pseudocysts, walledoff necrosis, and pancreatic fluid collections. Endosc Ultrasound 2016;5:215-7.

19. Ang TL, Kongkam P, Kwek AB, et al. A two-center comparative study of plastic and lumen-apposing large diameter self-expandable metallic stents in endoscopic ultrasound-guided drainage of pancreatic fluid collections. Endosc Ultrasound 2016;5:320-7.

20. Eliason K, Adler DG. Endoscopic ultrasound-guided transmural drainage of infected pancreatic necrosis developing 2 years after acute pancreatitis. Endosc Ultrasound 2015;4:260-5.

21. Guo J, Liu Z, Sun S, et al. Removal of displaced double flanged metal stent in walled-off necrosis by endoscopic ultrasonography. Endosc Ultrasound 2016;5:132-3. 